\title{
Heart failure-associated anemia: bone marrow dysfunction and response to erythropoietin
}

\author{
Willem-Peter T. Ruifrok • Cheng Qian • Herman H. W. Silljé • Harry van Goor • \\ Dirk J. van Veldhuisen • Wiek H. van Gilst • Rudolf A. de Boer
}

Received: 19 August 2010/Revised: 9 November 2010/Accepted: 7 December 2010 /Published online: 30 December 2010

(C) The Author(s) 2010. This article is published with open access at Springerlink.com

\begin{abstract}
Heart failure (HF)-associated anemia is common and has a poor outcome. Because bone marrow (BM) dysfunction may contribute to HF-associated anemia, we first investigated mechanisms of BM dysfunction in an established model of HF, the transgenic REN2 rat, which is characterized by severe hypertrophy and ventricular dilatation and SD rats as controls. Secondly, we investigated whether stimulation of hematopoiesis with erythropoietin (EPO) could restore anemia and BM dysfunction. After sacrifice, erythropoietic precursors (BFU-E) were isolated from the $\mathrm{BM}$ and cultured for 10 days. BFU-E were quantified and transcript abundance of genes involved in erythropoiesis were assayed. Number of BFU-E were severely decreased in BM of REN2 rats compared to SD rats $(50 \pm 6.2$ vs. $6.4 \pm 1.7, p<0.01)$. EPO treatment increased hematocrit in the SD-EPO group (after 6 weeks, $49 \pm 1$
\end{abstract}

Willem-Peter T. Ruifrok and Cheng Qian contributed equally to this work.

Electronic supplementary material The online version of this article (doi:10.1007/s00109-010-0710-6) contains supplementary material, which is available to authorized users.

W.-P. T. Ruifrok • C. Qian • H. H. W. Silljé

D. J. van Veldhuisen - W. H. van Gilst $\cdot$ R. A. de Boer $(\bowtie)$

Department of Cardiology, University Medical Center Groningen,

University of Groningen,

Hanzeplein 1,

9713 GZ Groningen, The Netherlands

e-mail: r.a.de.boer@thorax.umcg.nl

H. van Goor

Department of Pathology, University Medical Center Groningen,

University of Groningen,

Hanzeplein 1,

9713 GZ Groningen, The Netherlands vs. $58 \pm 1 \%, p<0.01$ ); however, in the mildly anemic REN2 rats, there was no effect ( $43 \pm 1$ vs. $44 \pm 1 \%$ ). This was paralleled by a $67 \%$ decrease in BFU-E in BM of REN2 rats compared to SD $(p<0.01)$. EPO significantly improved BFU-E in both SD and REN2 but could not restore this to control levels in the REN2 rats. Expression of several genes involved in differentiation (LMO2), mobilization (SDF-1), and iron incorporation (transferrin receptor) of the BM were differentially expressed in REN2 rats compared to SD rats, and EPO did not normalize this. Altogether, these results suggest that BM dysfunction is an important contributor to HF-associated anemia and that EPO is not an effective agent to treat $\mathrm{HF}$-associated anemia.

Keywords Anemia $\cdot$ Heart failure $\cdot$ Bone marrow dysfunction $\cdot$ REN2 Erythropoietin $\cdot$ Renin .

Renin-angiotensin system

\section{Introduction}

Anemia as a co-morbidity in heart failure (HF) is associated with a poor outcome $[1,2]$. The prevalence of anemia in $\mathrm{HF}$ is reported to be over $40 \%$ in patients with advanced HF [3]. The causes of anemia in HF are only partially understood, although several mechanisms have been implicated [4], including treatment with ACE-inhibitors [5], a blunted erythropoietin (EPO) production due to renal dysfunction [6], congestion [7], and iron deficiency [8]. However, these causes only partially explain the severity of anemia and are difficult to target.

Recent publications pointed towards bone marrow (BM) dysfunction as a potential contributing factor to anemia in HF. Iversen et al. showed decreased hematopoiesis in BM 
in an experimental (murine) model of post-myocardial infarction HF [9]. Our group recently provided the first clinical proof for the existence of BM dysfunction in human ischemic HF [10]. We showed that HF is associated with a profound and general BM dysfunction, simultaneously affecting multiple hematopoietic lineages. Very recently, the group of Iversen provided further evidence that HF is associated with general BM dysfunction by showing that anemia occurs in both ischemic and non-ischemic murine HF models [11]. Apparently, the mechanisms of anemia in HF are dependent of etiology and are possibly caused by general HF factors like overproduction of neurohormones or hypoperfusion [11]. Importantly, data as to whether proerythropoietic therapy could restore anemia and BM function in HF is lacking.

Therefore, we evaluated the severity and mechanisms of anemia and BM dysfunction in an established experimental model of hypertensive HF, the transgenic TGR(mREN2)27 rat. Furthermore, we investigated if stimulation of hematopoiesis with EPO administration could restore, at least in part, HF-associated anemia and BM function. Finally, we studied in detail well-established factors of erythroid differentiation (LMO2, GATA-1) and mobilization (MMP9, SDF-1) (Fig. 1, adapted from Koury et al. [12]). LMO2 and GATA-1 are both differentiation factors, where MMP9 mobilizes erythroid precursors from the BM into the peripheral blood, and SDF-1 is a negative regulator of erythroid mobilization. Furthermore, we analyzed factors involved in iron incorporation in the BM (transferrin receptor and ferroportin, both involved in maintaining cellular iron homeostasis) and studied if expression of these factors is changed during EPO administration.

\section{Materials and methods}

Animal model

We employed 6-week-old, male, homozygous TGR (mREN2)27 rats [13]. See "Electronic supplementary material" for detailed description. Rats were fed with ad libitum regular chow that contains sufficient amounts of vitamin B and iron (RMH-B 2181, AB Diets, The Netherlands). Over the course of the experiment, the mortality rate of REN2 rats was $20 \%$ (as reported; mortality was $0 \%$ for control (Sprague-Dawley, SD) rats). The experimental protocol was approved by the Animal Ethical Committee of the University of Groningen, The Netherlands, and conducted in accordance with existing guidelines for the care and use of laboratory animals.

Experimental protocol

To stimulate erythropoiesis, rats were randomized to treatment with the long-acting EPO analogue darbepoetin alpha $\left(40 \mu \mathrm{g} / \mathrm{kg}\right.$, Aranesp ${ }^{\circledR}$, Amgen Inc., Thousand Oaks, CA, USA) administered subcutaneously every 3 weeks (baseline, 3 and 6 weeks) or saline (used as control). The dose of EPO was based on our extensive experience with this compound and previous data that this dose effectively raises hematocrit in SD rats [14-19]. Rats were sacrificed at week 7 of the experimental protocol (when aged 13 weeks). In total, four groups were studied: SD-PL (placebo; $N=12$ ), REN2-PL $(N=12)$, SD-EPO $(N=12)$, REN2-EPO $(N=15)$. At baseline, 3 and 6 weeks, blood was drawn from the tail vein for a full blood count. At sacrifice, we analyzed serum
Fig. 1 Overview of different factors involved in differentiation and mobilization during different stages of erythropoiesis, which expression was measured (results shown in Fig. 5). Adapted from Koury et al. [12], according to Hanson et al. [29], Ohneda et al. [31], and Jin et al. [32]. Factors highlighted in green are positive regulators, factor highlighted in red is a negative regulator. $H S C$ hematopoietic stem cell, $B F U-E$ burst forming unit-erythroid, $C F U-E$ colony forming uniterythroid, $R E T$ reticulocyte, $R B C$ red blood cell, LMO2 LIM domain only 2 protein, TAL T cell acute lymphocytic leukemia protein, GATA GATA binding protein, $F O G$ friend of GATA, $M M P$ matrix metallopeptidase, $S D F$ stromal cell-derived factor

\section{Stages of erythroid differentiation}

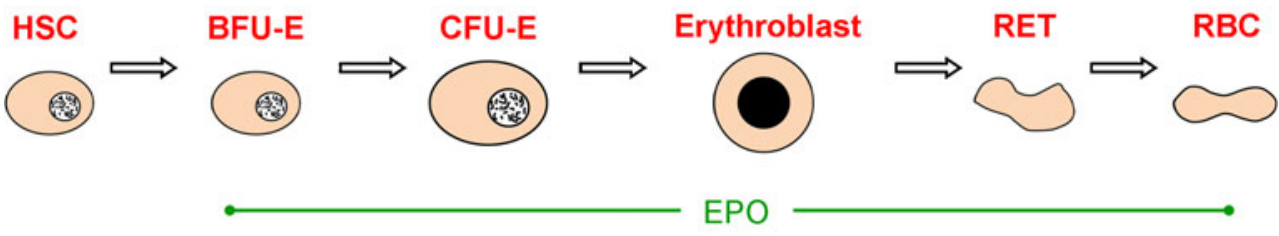

1. Factors involved in differentiation of erythroid bone marrow cells

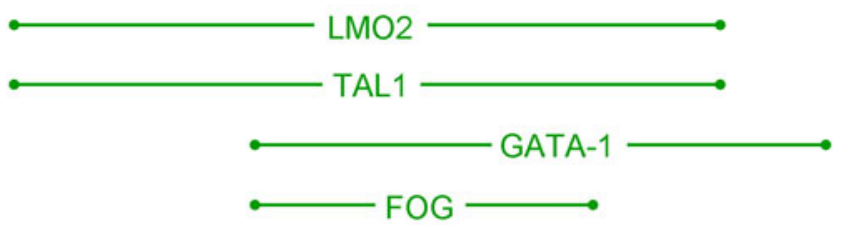

2. Factors for mobilization of erythroid bone marrow cells

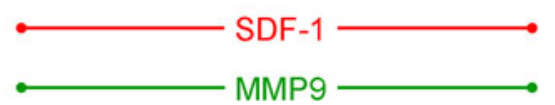


iron status. Blood pressure via tail cuff and hemodynamic data at sacrifice were measured as described [20, 21]. Furthermore, to monitor the development of HF, echocardiography was performed at baseline, 3 and 6 weeks. For details, see "Electronic supplementary material".

\section{Bone marrow isolation and MACs sorting}

BM was isolated from tibia and femurs as previously described [22]. C-kit positive (c-kit ${ }^{+}$) cells were isolated as described in the "Electronic supplementary material". Briefly, the cell pellet was washed twice with PBS to remove platelets. Cells were labeled with polyclonal rabbit anti c-kit antibody, followed by goat anti-rabbit $\operatorname{IgG}$ microbeads. Anti c-kit-labeled cells were sorted by using mini-magnetic activated cell sorting columns in a magnetic field. Effluent was collected in chilled IMEM medium. C$\mathrm{kit}^{+}$cells were collected with $1 \mathrm{~mL}$ chilled IMEM medium, and cell number was counted.

\section{Cell proliferation assay}

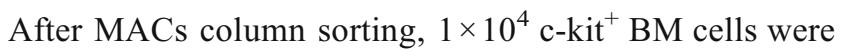
plated in $1 \mathrm{~mL}$ semi-solid medium (MethoCult, Stem Cell Technologies, Köln, Germany) in $35 \mathrm{~mm}$ Petri dishes in duplicate. The dishes were incubated at $37 \mathrm{C}$ with $5 \%$ $\mathrm{CO}_{2}$ and $95 \%$ humidity for 2 weeks. Colonies were scored using an inverted phase-controlled microscope (Leica 090-135002, Leica Microsystems, CMS GmbH, Germany). Burst-forming units-erythroid (BFU-E) were defined as consisting of 30 or more cells. Colony-forming units (CFU) were defined as consisting of 50 or more cells.

\section{FACS analysis}

BM mononuclear cells were harvested from tibia and femur of experimental animals and frozen in liquid nitrogen with $10 \%$ DMSO in medium in assay. FACS analysis was performed on an LSRII cytometer (BD Biosciences, San Jose, CA, USA). For phenotypic analysis by flow cytometry, $2-5 \times 10^{7}$ cells were dissolved in $10 \mathrm{~mL}$ cold DMEM with $10 \%$ FBS. Cell pellet were resuspended in $80 \mu \mathrm{L}$ PBS/ $0.5 \%$ BSA. Samples were subsequently stained with rabbit polyclonal anti c-kit antibody $\left(1 \mu \mathrm{g} / 10^{6}\right.$ cells, Santa Cruz Biotechnology, Heidelberg, Germany) on ice for $30 \mathrm{~min}$. After washing twice with $2 \mathrm{~mL} \mathrm{PBS} / 0.5 \%$ BSA, cells were incubated with Alexa Fluor ${ }^{\circledR} 647\left(1 \mu \mathrm{g} / 10^{6}\right.$ cells, Invitrogen, OR, USA) and FITC rat anti-mouse IgG $\left(1 \mu \mathrm{g} / 10^{6}\right.$ cells, BD Biosciences Pharmingen, Breda, The Netherlands) as secondary antibodies. Cells were then suspended in $0.5 \mathrm{~mL}$ PBS $/ 0.5 \%$ BSA and analyzed within $1 \mathrm{~h}$. Unstained cells and cells stained with second antibody only were used as a negative control. Data were analyzed by FlowJo software (V7.5.5, Ashland, OR, USA).

Quantitative real-time PCR

Total RNA from c-kit ${ }^{+}$cells and left ventricular (LV) tissue was extracted using the Nucleospin RNA II kit, according to manufacturer's protocol (Machery-Nagel, Düren, Germany). cDNA synthesis and quantitative realtime PCR (RT-qPCR) were performed as previously described with using $0.5 \mu \mathrm{g}$ total RNA [23]. mRNA levels were expressed in relative units based on a standard curve obtained with serial dilutions of a calibrator cDNA mixture. To normalize expression data, multiple reference genes were used as described [24]. Please see "Electronic supplementary material" for full protocol and list of primers.

\section{Statistical analyses}

Results are reported as means \pm standard errors of the mean. Number of BFU-E, CFU, and c-kit ${ }^{+}$cells were normalized to SD-PL group. Statistical analysis among groups was performed by ANOVA with the Bonferroni post hoc test if distributed normally or with the Kruskall-Wallis test followed by Mann-Whitney $U$ test when skewed distributed. All $p$ values are two-tailed, and a $p$ value of less than 0.05 was considered significant. All analyses were performed using SPSS version 16.0 software (SPSS, Chicago, IL, USA).

\section{Results}

Hypertensive REN2 rats develop heart failure

REN2 and SD rats were treated with or without EPO and were monitored for 6 weeks and sacrificed at an age of 13 weeks. As expected, REN2 rats suffered from severe hypertension (Fig. 2), providing the substrate for HF development. Heart weight (adjusted for tibia length), LV end diastolic pressure (LVEDP), and atrial natriuretic peptide (ANP) mRNA expression were all significantly increased in the REN2-PL and REN2-EPO groups, indicative for HF (Fig. 2). Table 1 shows the hemodynamic data at sacrifice. Indices of maximal contraction and relaxation of the LV (dPdtmax and dPdtmin) were both significantly decreased in the REN2 groups, compared to the SD groups, indicating development of HF. Cardiac output (CO), assessed with echo Doppler prior to sacrifice, is decreased in both REN2 groups compared to the SD groups. Echocardiographic data during the experiment confirmed development of LV hypertrophy and LV dilatation in REN2 rats (shown in "Electronic supplementary material"). 
Fig. 2 Shows that the used animal model is a model of hypertensive heart failure. a Mean arterial pressure (MAP) during the experiment. MAP is significantly higher at all time points for REN2 groups compared to SD groups. b Heart weight normalized to tibia length. c Left ventricular end diastolic pressure (LVEDP) at sacrifice. d mRNA expression of atrial natriuretic peptide (ANP) in left ventricular tissue. ANP is expressed as fold change. $H W$ heart weight, Base baseline, $W k$ week, $P L$ placebo. ${ }^{*} p<0.05 ; * * p<0.01$

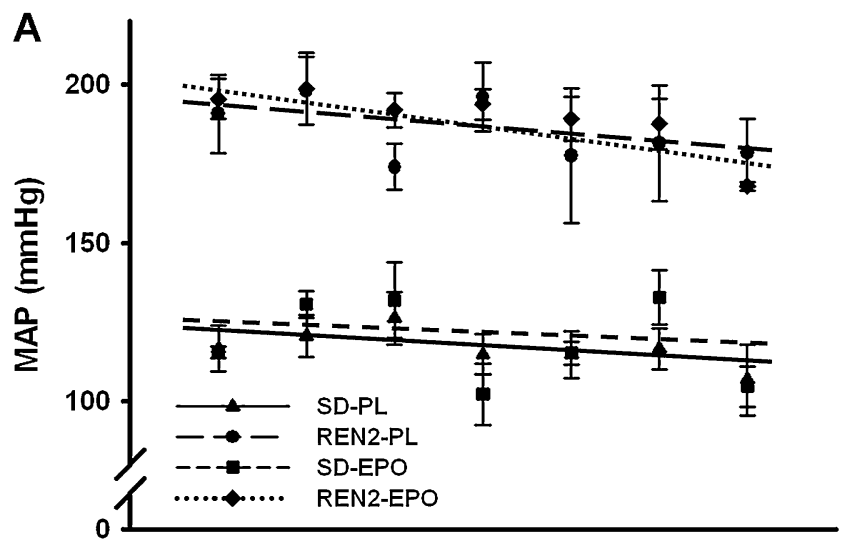

Base Wk 1 Wk 2 Wk 3 Wk 4 Wk 5 Wk 6

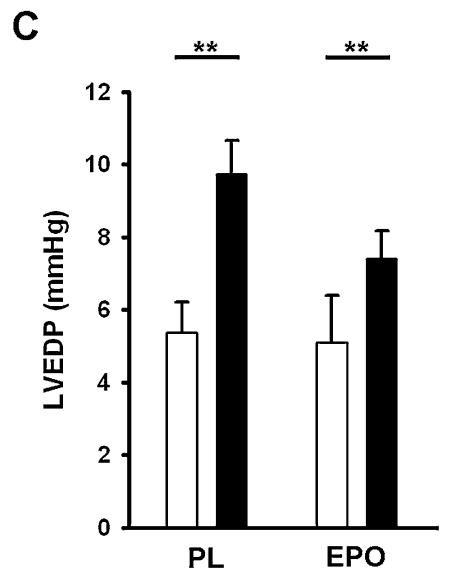

B

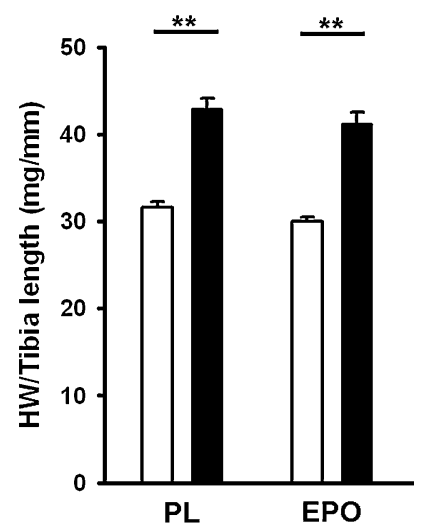

D
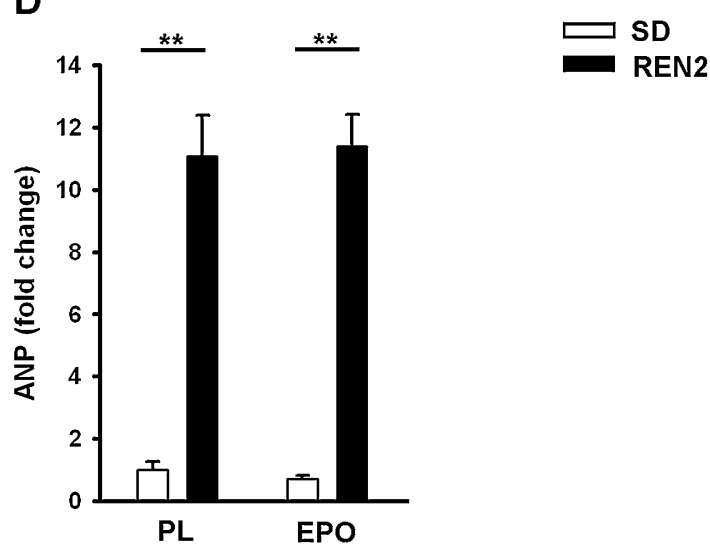

REN2 rats are anemic and have a limited response to EPO

Table 2 shows the full blood count for all experimental groups at sacrifice. As hypothesized, REN2 rats were mildly anemic and had mild to moderate reductions of other hematologic parameters as well (except platelet and reticulocyte count, which were increased). Administration of EPO increased hematocrit $(\mathrm{Ht})$ levels significantly in the SD-EPO group compared to the SD-PL group (57.8 \pm $1.1 \%$ vs. $49.3 \pm 0.8 \%, p<0.01)$; however, EPO administration did only marginally increase Ht in the REN2-EPO group $(44.0 \pm 0.6 \%$ vs. $42.7 \pm 0.5 \%$, compared to REN2-
PL, $p=$ NS, Fig. 3). An identical pattern was observed for hemoglobin levels. Furthermore, RBC, MCV, and $\mathrm{MCH}$ were all significantly decreased in both the REN2-PL and the REN2-EPO groups, providing further evidence of anemia. We observed an increased reticulocyte count in the REN2 groups, which is commonly seen in anemia. EPO plasma levels increased significantly in all EPO treated groups (measured with ELISA as before [17], see "Electronic supplementary material"). Deficiencies of iron, vitamin B12, and folic acid were ruled out and did not differ between SD and REN2 groups (see "Electronic supplementary material"').
Table 1 Data at sacrifice

Table shows weights and hemodynamic data at sacrifice. Cardiac output (CO) was assessed with echo Doppler at sacrifice in anesthetized animals.

$L V$ left ventricle, $T L$ tibia length, $P L$ placebo.

$* p<0.05$ vs. SD-PL; $* * p<0.05$ vs. SD-EPO

\begin{tabular}{lcccc}
\hline & SD-PL $(N=12)$ & REN2-PL $(N=12)$ & SD-EPO $(N=12)$ & REN2-EPO $(N=15)$ \\
\hline LV weight (mg) & $841 \pm 15$ & $1,105 \pm 44^{*}$ & $809 \pm 17$ & $1,157 \pm 36^{* *}$ \\
TL (mm) & $38.3 \pm 0.3$ & $38.1 \pm 0.2$ & $38.6 \pm 0.2$ & $38.2 \pm 0.3$ \\
Body weight (g) & $367 \pm 6$ & $309 \pm 7^{*}$ & $360 \pm 8$ & $316 \pm 9^{* *}$ \\
Heart rate (bpm) & $352 \pm 17$ & $347 \pm 12$ & $352 \pm 12$ & $350 \pm 10$ \\
dPdtmax (mmHg/s) & $7,993 \pm 290$ & $5,625 \pm 356^{*}$ & $8,676 \pm 618$ & $5,785 \pm 295^{* *}$ \\
dPdtmin (mmHg/s) & $-9,211 \pm 666$ & $-5,730 \pm 335^{*}$ & $-10,249 \pm 711$ & $-6,010 \pm 367^{* *}$ \\
CO (mL/min) & $145 \pm 4$ & $112 \pm 10^{*}$ & $138 \pm 6$ & $121 \pm 11^{* *}$ \\
\hline
\end{tabular}


Table 2 Hematology

\begin{tabular}{|c|c|c|c|c|}
\hline & SD-PL $(N=12)$ & REN2-PL $(N=12)$ & SD-EPO $(N=12)$ & REN2-EPO $(N=15)$ \\
\hline $\mathrm{Hb}(\mathrm{mmol} / \mathrm{L})$ & $9.6 \pm 0.8$ & $9.0 \pm 0.1$ & $12.4 \pm 0.2 *$ & $9.3 \pm 0.1 * * *$ \\
\hline Ht $(\%)$ & $49.3 \pm 0.8$ & $42.7 \pm 0.5^{*}$ & $57.8 \pm 1.1^{*}$ & $44.0 \pm 0.6^{* * *}$ \\
\hline WBC $\left(\times 10^{9} / \mathrm{L}\right)$ & $14.6 \pm 0.5$ & $11.4 \pm 0.3^{*}$ & $14.8 \pm 0.6$ & $11.6 \pm 0.5^{* * *}$ \\
\hline $\mathrm{RBC}\left(\times 10^{12} / \mathrm{L}\right)$ & $8.8 \pm 0.2$ & $8.0 \pm 0.1^{*}$ & $9.8 \pm 0.2^{*}$ & $8.0 \pm 0.1^{* * *}$ \\
\hline PLT $\left(\times 10^{9} / \mathrm{L}\right)$ & $522 \pm 32$ & $744 \pm 33 *$ & $485 \pm 36$ & $711 \pm 25^{* * *}$ \\
\hline RET (promille) & $27.5 \pm 6.1$ & $31.1 \pm 2.4^{*}$ & $7.0 \pm 1.4^{*}$ & $33.2 \pm 2.7 * * *$ \\
\hline MCV (fl) & $57.0 \pm 0.7$ & $53.2 \pm 0.3 *$ & $58.2 \pm 1.2$ & $54.8 \pm 0.4 * * ; * * *$ \\
\hline $\mathrm{MCH}$ (atto mol) & $1,204 \pm 8$ & $1,127 \pm 12^{*}$ & $1,247 \pm 20^{*}$ & $1082 \pm 83^{* * *}$ \\
\hline $\mathrm{MCHC}(\mathrm{mmol} / \mathrm{L})$ & $21.1 \pm 0.2$ & $21.2 \pm 0.2$ & $21.5 \pm 0.2$ & $22.2 \pm 1.0$ \\
\hline
\end{tabular}

Table shows full blood count at sacrifice.

$H b$ hemoglobin, $H t$ hematocrit, $W B C$ white blood cell count, $R B C$ red blood cell count, PLT platelet count, RET reticulocytes, $M C V$ mean corpuscular volume, $M C H$ mean corpuscular hemoglobin, $M C H C$ mean corpuscular hemoglobin concentration, $P L$ placebo.

${ }^{*} p<0.05$ vs. SD-PL; $* * p<0.05$ vs. REN2-PL; $* * * p<0.05$ vs. SD-EPO
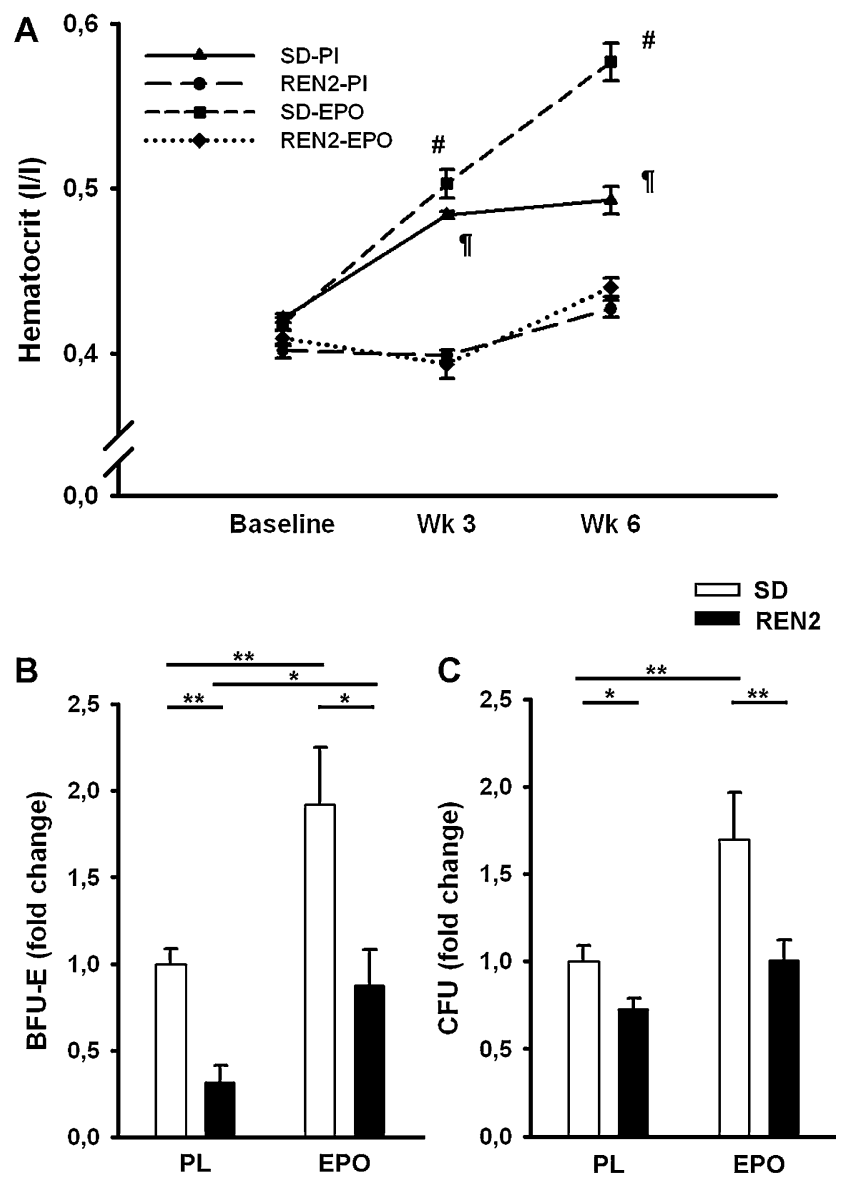

Fig. 3 a Change in hematocrit $(\mathrm{Ht})$ levels during experiment. EPO administration cannot increase Ht levels in REN2 rats. b Number of burst forming units-erythroid (BFU-E, normalized to SD-PL). c Number of colony forming units (CFU, normalized to SD-PL). $P L$ placebo, $B M$ bone marrow, $W k$ week, $\# p<0.05$ vs. SD-PL, REN2-PL and REN2-EPO, $q p<0.05$ vs. REN2-PL and REN2-EPO, ${ }^{*} p<0.05$, $* * p<0.01$

Bone marrow dysfunction in the REN2 rats

To provide mechanistic insight into the causes of anemia, we studied the number of BFU-E and CFU colonies with a cell proliferation assay (Fig. 3b, c). REN2 rats (treated with placebo) had a severely decreased number of BFU-E colonies, compared to SD-PL $(0.33 \pm 0.11$-fold, normalized to SD-PL, $p<0.05$ ). This indicates either a reduced number or a limited proliferation capacity of erythroid precursors in the BM. EPO administration increased the number of BFU-E colonies, both in the SD-EPO group $(1.92 \pm 0.33$-fold vs. SD$\mathrm{PL}, p<0.05)$ as well as in the REN2-EPO group, albeit much less $(0.88 \pm 0.21$-fold vs. SD-PL, $p<0.05)$.

To dissect abnormalities in the erythroid precursors only from general abnormalities in the BM, we also investigated number of CFU colonies. Number of CFU is only slightly, but significantly decreased in the REN2-PL group compared to SD-PL group.

Low levels of hematopoietic stem cells in REN2 rats

To confirm the findings in the cell proliferation assay, we performed FACS analysis of c-kit ${ }^{+}$cells in BM (Fig. 4). Compared to SD-PL, the number of c-kit ${ }^{+}$cells is reduced in REN2-PL (with $43 \%, p<0.05$ ). In line with the BFU-E cell proliferation assay number, EPO treatment increased the number of c-kit ${ }^{+}$cells measured by FACS in both the SD-EPO group (1.6-fold, $p<0.05$ vs. SD-PL) and the REN2-EPO group (to baseline, vs. SD-PL, $p=\mathrm{NS}$ ). However, the response to EPO was less strong in the REN2 rats.

mRNA expression of factors involved in erythroid differentiation, mobilization, and iron incorporation

LMO2 was significantly reduced in the REN2-PL group $(34 \%$ vs. SD-PL, $p<0.05)$. EPO treatment reduced the 

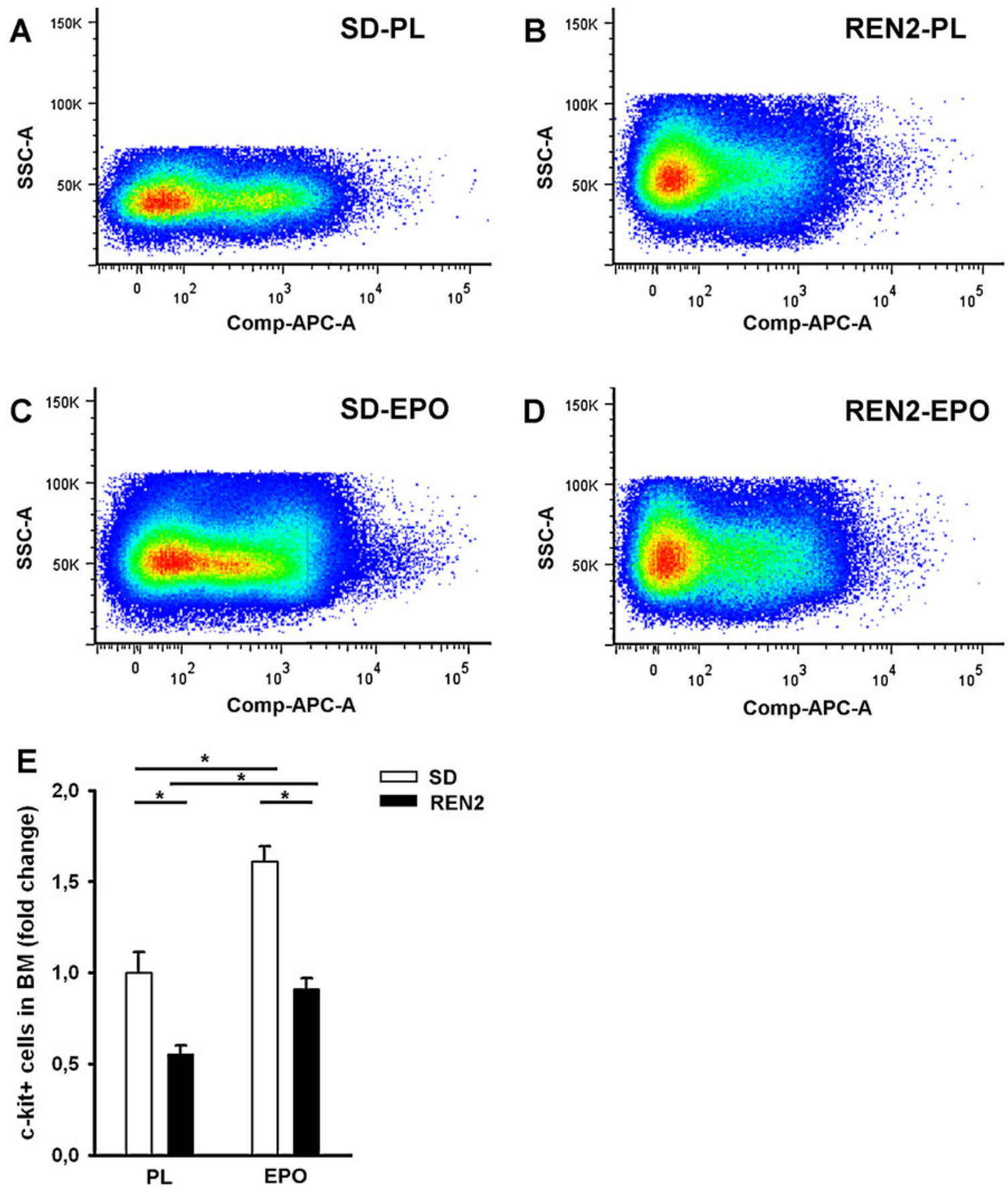

Fig. 4 a-d Typical examples of FACS analysis. Every dot is a single cell count. The $x$-axis (Comp-APC-A) is the gate for fluorescent cells, the $y$-axis (SSC-A) is the cell size. Red color is high density of cell

count; blue color is lower density of cell count. e Number of the erythroid c-kit ${ }^{+}$cells in bone marrow. $P L$ placebo, $B M$ bone marrow. ${ }^{*} p<0.05 ;{ }^{* *} p<0.01$

LMO2 expression in the SD-EPO group but did not increase the LMO2 expression in the REN2-EPO group. Vice versa, expression of GATA-1 was 2.3-fold increased in the REN2-PL group compared to the SD-PL group ( $p<$ 0.01). Treatment with EPO increased the GATA-1 expression in the SD-EPO group, but did not affect the GATA-1 expression in the REN2-EPO group. These data suggest that differentiation of erythrocyte-like BM precursors is

inhibited in HF and that EPO treatment partially restores this (Fig. 5).

Expression of MMP9 was reduced with $60 \%$ in the REN2-PL group compared to the SD-PL group $(p<0.05)$. Treatment with EPO did not exert substantial effects on MMP9 expression in neither SD nor REN-2 rats. In contrast, SDF-1 expression was significantly increased in the REN2-PL group (3.5-fold vs. SD-PL, $p<0.05$ ). Treat- 
Fig. 5 mRNA expression of different factors involved in differentiation $(\mathbf{a}, \mathbf{b})$ and mobilization (c, d) of c-kit ${ }^{+}$cells. mRNA expression of different factors involved in iron metabolism (e, f). $L M O 2$ LIM domain only 2 protein, GATA- 1 GATA binding protein, $M M P 9$ matrix metallopeptidase, $S D F-1$ stromal cellderived factor, $P L$ placebo. ${ }^{*} p<0.05 ; * * p<0.01$
A

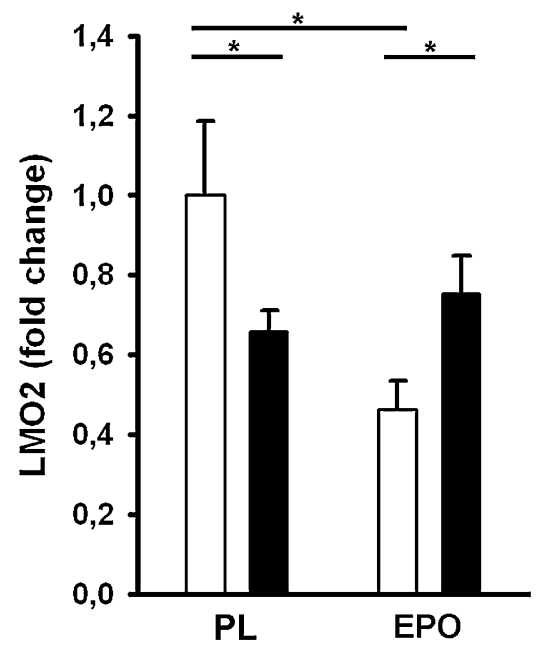

B

SD

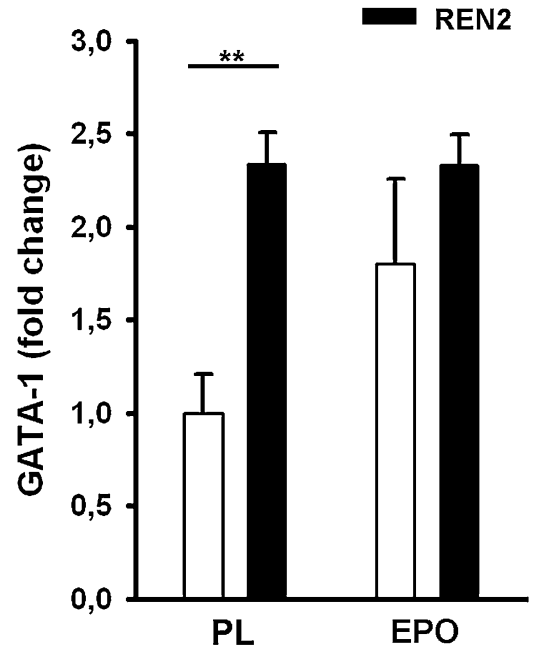

C

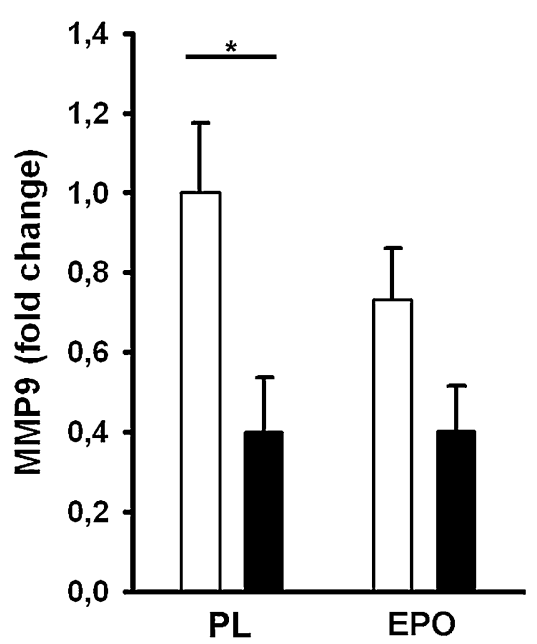

D

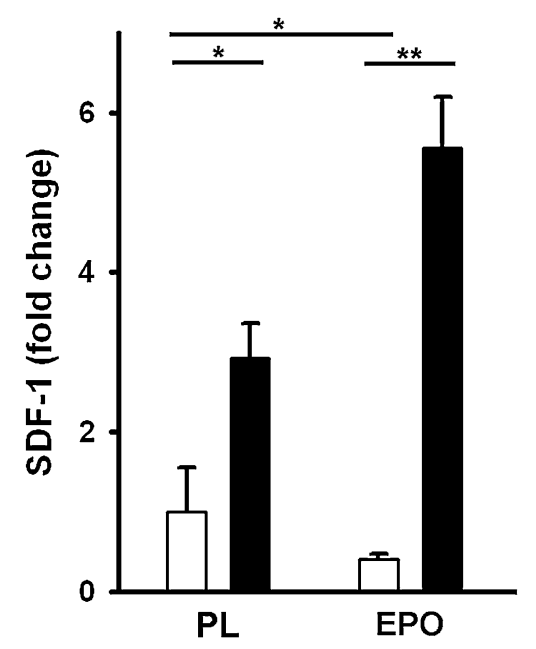

$\mathbf{F}$

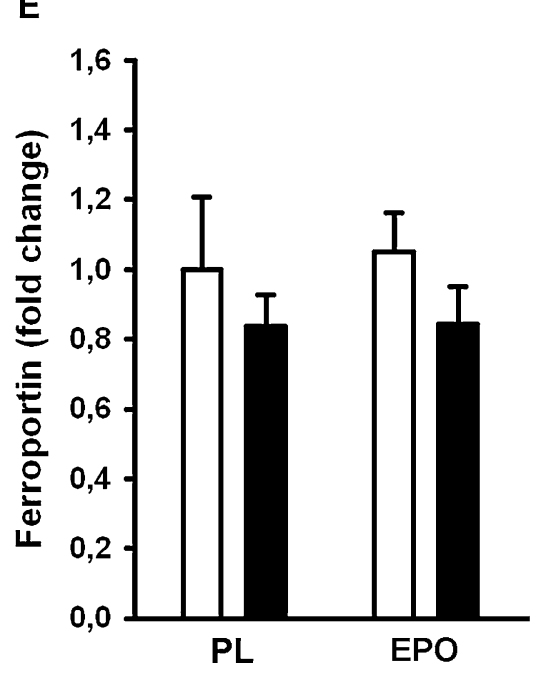

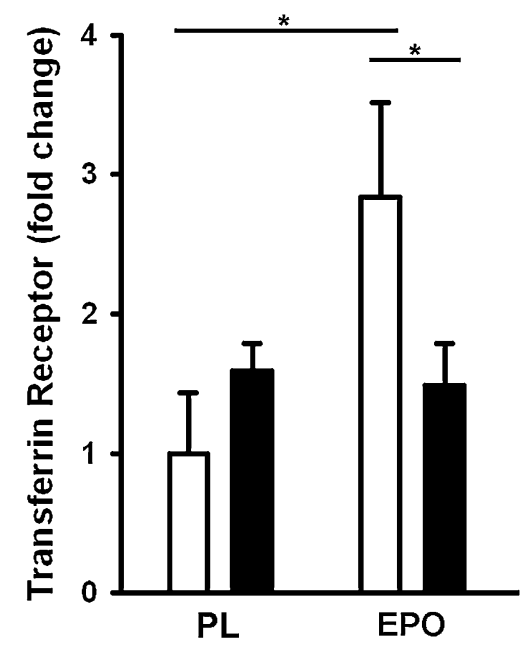


ment with EPO further increased the expression of SDF-1 (5.6-fold vs. SD-EPO, $p<0.01$ ). These data suggest that mobilization of erythrocyte-like BM precursors is inhibited in HF and that this cannot be restored by EPO treatment.

mRNA expression of ferroportin was not statistically different between the four groups, albeit that ferroportin is slightly less expressed in both REN2 groups. mRNA expression of transferrin receptor was comparable between the SD-PL vs. REN2-PL groups (1.6 \pm 0.2 -fold vs. SD-PL, $p=\mathrm{NS})$. However, after treatment with EPO, the expression of transferrin receptor was increased in the SD-EPO group (2.8 \pm 0.7 -fold vs. SD-PL, $p<0.05$ ), where the expression was not increased in the REN2-EPO group, indicating a reduction in iron incorporation in the erythroid cells in REN2 rats after EPO administration.

\section{Discussion}

In hypertensive REN2 rats with progressive HF, we observed mild anemia and a profound dysfunction of the erythroid lineage in the BM. Treatment with EPO with the aim to rescue this anemia did not diminish the anemia. Apparently, HF-associated BM dysfunction is a rather complex phenomenon that may require other treatment options. In concert with this, we observed that along the entire line of maturation of the erythroid lineage, multiple genes associated with differentiation, mobilization, and iron incorporation were abnormally expressed in BM from HF rats. Figure 6 provides an overview of the mechanisms of BM dysfunction involved in HF-associated anemia.

We used a monogenetic rat model with overexpression of the renin gene, resulting in hypertension and concomitant HF development. These rats also develop mild anemia, which is in line with the observations of Iversen et al., who described for the first time the existence of HF-associated anemia in mice [9]. Our results extend this observation of Iversen to another species (rat) and moreover show that this also occurs in animals in which hypertension is the sole source for HF development.

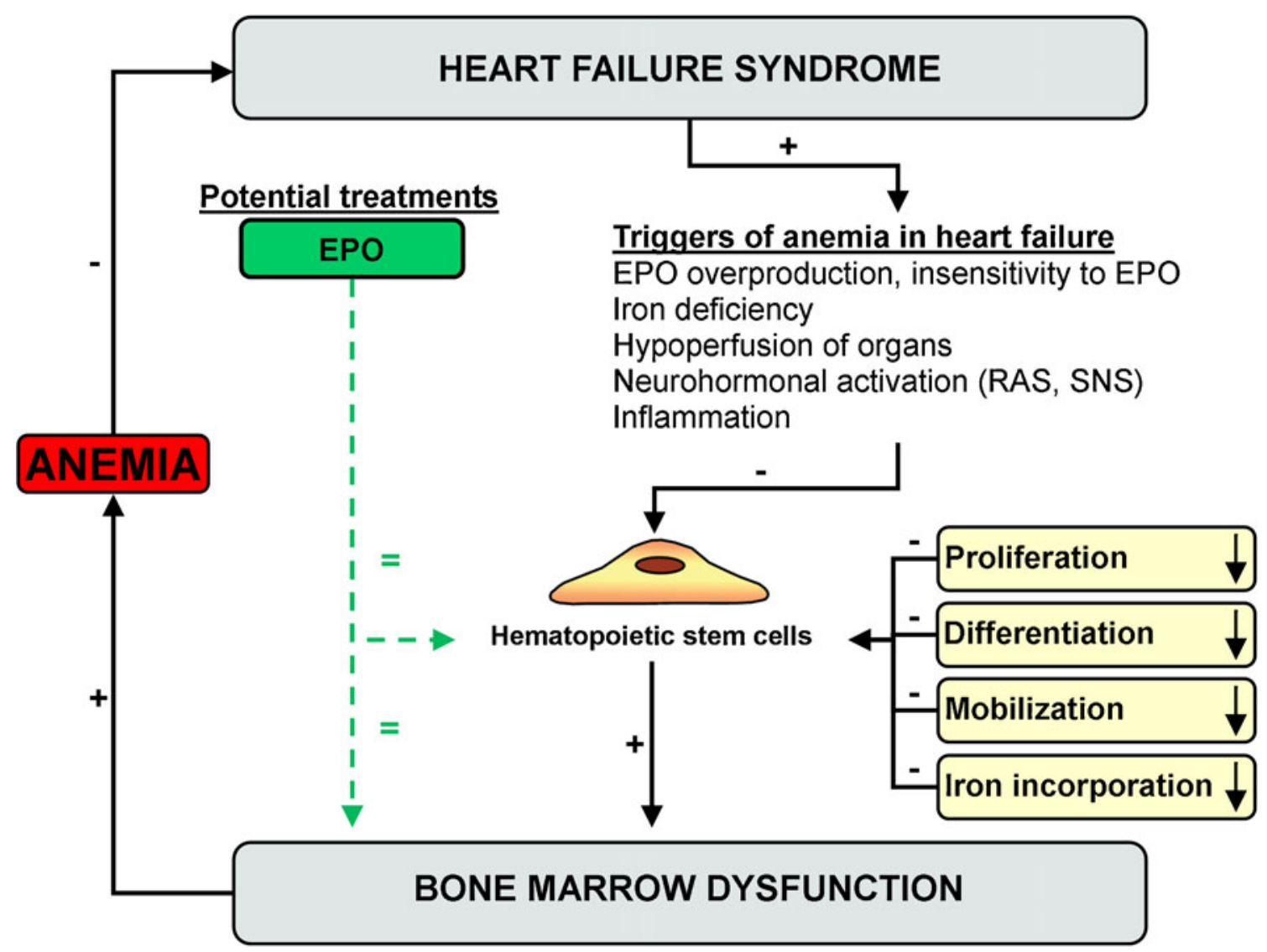

Fig. 6 Schematic overview of the mechanisms of bone marrow dysfunction involved in HF-associated anemia. Different triggers of anemia in heart failure such as insensitivity of EPO, iron deficiency, and neurohormonal activation have a negative effect on the hematopoietic stem cells in the bone marrow. Furthermore, impaired proliferation, differentiation, mobilization, and iron incorporation in hematopoietic stem cells contribute to the bone marrow dysfunction, causing anemia and adding to the heart failure syndrome, which in turn triggers anemia in heart failure, etc. EPO treatment does not rescue the dysfunction of the erythroid lineage and does not improve heart failure-associated anemia. SNS sympathetic nervous system, $R A S$ renin-angiotensin system 
Since HF-associated anemia has a dramatic impact on prognosis in humans [1-3, 25], we tried to restore this by EPO treatment. As expected, EPO treatment resulted in a clear increase in $\mathrm{Ht}$ in the control rats. Surprisingly, however, EPO did not rescue anemia in the REN2 rats and only showed marginal effects on $\mathrm{Ht}$ values. Since the main action of EPO is the regulation of erythropoiesis, this is apparently not the single culprit in HF-associated anemia. Several clinical observations from our group suggested that EPO levels in HF are disconcordant with the (expected) levels of hemoglobin [26-28], and our current observations that EPO cannot rescue $\mathrm{HF}$-associated anemia are in concert with this. This misbalance between EPO and hemoglobin levels is related to an increased mortality risk. Mechanistically, this may be explained by a diminished response of the BM to high plasma EPO levels in HF, a phenomenon we confirm in our present study. Together, this indicates that in HF-associated anemia other treatment options will be needed.

To obtain further insight in the cause of this anemia, we analyzed the BM. We observed a profound (43\%) decrease in BM hematopoietic progenitor cells (c-kit ${ }^{+}$cells) in REN2 rats as compared to control animals. This is in line with the observation of Iversen et al., who observed a reduction of up to $60 \%$ of similar CD34+ cells in mice with HF [9]. Although not investigated in our rat model, they suggested that the mechanisms of HF-associated anemia may depend on the etiology of the cardiac disease, where inflammation seems to be dominant in ischemic HF, while neurohormonal factors seem to be the player in non-ischemic HF [11].

To further characterize BM dysfunction and the irresponsiveness to EPO in the REN2 rat model of HF, we performed RT-qPCR analysis to measure the expression of markers of differentiation (LMO2, GATA-1) and mobilization (MMP9, SDF-1) of the erythroid lineage and incorporation of iron in these cells (ferroportin, transferrin receptor) (Fig. 5). LMO2, a positive regulator of erythroid differentiation [29], was down-regulated in the REN2-PL group, which may contribute to the decreased number of BFU-E. GATA-1 is a transcription factor playing a key role in gene regulation during erythroid differentiation [30]. This factor is strictly controlled during development and differentiation of erythroid cells and essential for the survival of erythroid progenitors [31]. GATA-1 mRNA expression is 2.3-fold increased in our experiments in the REN2-PL group, indicating that survival of the erythroid progenitors is promoted in this weakened erythropoietic system.

Furthermore, impaired mobilization of erythroid progenitors from the BM could contribute to the lack of increase in Ht upon EPO stimulation. Degradation of BM SDF-1 by MMP9 contributes to increased mobilization of erythroid progenitors [32]. So, decreased levels of MMP9, which result in increased levels of BM SDF-1, could in turn lead to impaired mobilization of erythroid progenitors from the BM in REN2 rats.

Analysis of factors involved in iron transportation yielded some interesting results. mRNA expression of ferroportin, involved in iron transportation from inside of a cell to the outer surface, was not different between any group. mRNA expression of transferrin receptor, involved in maintaining cellular iron homeostatis, was comparable between the SD-PL vs. REN2-PL groups. However, after treatment with EPO, expression was increased in the SDEPO group, where expression was not increased in the REN2-EPO group. We speculate that the lower expression of transferrin receptor in REN2 rats treated with EPO is associated with lesser iron incorporation in erythroid cells, which may explain in part the lesser response to EPO. In general, we observed a changed expression profile of genes involved in differentiation, mobilization, and incorporation of iron in BM.

Besides defects in the erythropoietic lineage of the BM (reduction in c-kit ${ }^{+}$cells and BFU-E), we also observed a reduction in number of $\mathrm{CFU}$ (with 27\%) compared to control animals. EPO treatment did not improve these values in the REN2 rats. We recently showed that human $\mathrm{HF}$ is associated with a profound and general BM dysfunction, simultaneously affecting multiple hematopoietic lineages [10]. Since all these patients obtained optimal HF-treatment and were obviously more heterogeneous, our confirmation in this monogenetic rat model further confirms these observations in a human population and show that it is treatment independent. Moreover, HF-associated anemia appears to be a general phenomenon in mammals.

dPdtmax, dPdtmin, and $\mathrm{CO}$ were all significantly decreased in the REN2 groups compared to the SD groups, indicating development of HF. Hypoperfusion of the bone marrow by reduced $\mathrm{CO}$ could potentially affect erythropoiesis in HF [8]. However, we believe that hypoperfusion is not (yet) a critical factor, as we have sacrificed the REN2 rats before overt (decompensated) HF had ensued.

Because the REN2 transgene is expressed ubiquitously and thus also in the hematopoietic cells, we cannot exclude that the effects of the transgene mediate the phenotype at least to some extent. However, the sequel of events in the development of HF in the REN2 rat (hypertrophy, diastolic dysfunction, systolic dysfunction, overt HF, death) are very much alike the human situation. Furthermore, the mild anemia and bone marrow dysfunction associated with HF in the REN2 rat are also comparable to what we observed in earlier human studies. So, although this model (like any rodent model) has limitations, the phenotype we study herein is, at least to a large extent, comparable to the human situation.

In conclusion, in REN2 rats with progressive HF, there is mild anemia and a profound dysfunction of the erythroid 
lineage of the BM. Response to EPO is diminished in REN2 rats and does not rescue the dysfunction of the erythroid lineage. Multiple genes appear to be involved in this process. This does, however, not exclude EPO as a treatment option in HF, because we and others have shown that EPO has also direct positive effects on the heart, including anti-apoptotic, pro-angiogenic, and antiischemic actions [14-19]. Therefore, EPO can be beneficial in HF but does not improve HF-associated anemia. The latter probably needs a more complex treatment regimen.

Acknowledgements We thank Inge Baudoin, Bianca Meijeringh, and Linda van Genne for their expert technical assistance.

Funding This study was funded in part by grants from the Netherlands Organization for Scientific Research (VENI 016-106117) and Netherlands Heart Foundation (Grant 2007T046).

Conflicts of interest The authors declare no competing financial interests.

Open Access This article is distributed under the terms of the Creative Commons Attribution Noncommercial License which permits any noncommercial use, distribution, and reproduction in any medium, provided the original author(s) and source are credited.

\section{References}

1. Anand I, McMurray JJ, Whitmore J, Warren M, Pham A, McCamish MA, Burton PB (2004) Anemia and its relationship to clinical outcome in heart failure. Circulation 110:149154

2. O’Meara E, Clayton T, McEntegart MB, McMurray JJ, Lang CC, Roger SD, Young JB, Solomon SD, Granger CB, Ostergren J et al (2006) Clinical correlates and consequences of anemia in a broad spectrum of patients with heart failure: results of the Candesartan in Heart Failure: Assessment of Reduction in Mortality and Morbidity (CHARM) Program. Circulation 113:986-994

3. Groenveld HF, Januzzi JL, Damman K, van Wijngaarden J, Hillege HL, van Veldhuisen DJ, van der Meer P (2008) Anemia and mortality in heart failure patients: a systematic review and meta-analysis. J Am Coll Cardiol 52:818-827

4. Westenbrink BD, de Boer RA, Voors AA, van Gilst WH, van Veldhuisen DJ (2008) Anemia in chronic heart failure: etiology and treatment options. Curr Opin Cardiol 23:141-147

5. van der Meer P, Lipsic E, Westenbrink BD, van de Wal RM, Schoemaker RG, Vellenga E, van Veldhuisen DJ, Voors AA, van Gilst WH (2005) Levels of hematopoiesis inhibitor $N$-acetyl-serylaspartyl-lysyl-proline partially explain the occurrence of anemia in heart failure. Circulation 112:1743-1747

6. Pfeffer MA (2008) Anemia treatment in chronic kidney disease: shifting uncertainty. Heart Fail Rev 13:425-430

7. Westenbrink BD, Visser FW, Voors AA, Smilde TD, Lipsic E, Navis G, Hillege HL, van Gilst WH, van Veldhuisen DJ (2007) Anaemia in chronic heart failure is not only related to impaired renal perfusion and blunted erythropoietin production, but to fluid retention as well. Eur Heart J 28:166-171
8. Anker SD, Comin Colet J, Filippatos G, Willenheimer R, Dickstein K, Drexler H, Luscher TF, Bart B, Banasiak W, Niegowska J et al (2009) Ferric carboxymaltose in patients with heart failure and iron deficiency. N Engl J Med 361:2436-2448

9. Iversen PO, Woldbaek PR, Tonnessen T, Christensen G (2002) Decreased hematopoiesis in bone marrow of mice with congestive heart failure. Am J Physiol Regul Integr Comp Physiol 282: R166-R172

10. Westenbrink BD, Voors AA, de Boer RA, Schuringa JJ, Klinkenberg T, van der Harst P, Vellenga E, van Veldhuisen DJ, van Gilst WH (2010) Bone marrow dysfunction in chronic heart failure patients. Eur J Heart Fail 12:676-684

11. Iversen PO, Andersson KB, Finsen AV, Sjaastad I, von Lueder TG, Sejersted OM, Attramadal H, Christensen G (2010) Separate mechanisms cause anemia in ischemic vs. nonischemic murine heart failure. Am J Physiol Regul Integr Comp Physiol 298:R808R814

12. Koury MJ, Sawyer ST, Brandt SJ (2002) New insights into erythropoiesis. Curr Opin Hematol 9:93-100

13. de Boer RA, Pokharel S, Flesch M, van Kampen DA, Suurmeijer AJ, Boomsma F, van Gilst WH, van Veldhuisen DJ, Pinto YM (2004) Extracellular signal regulated kinase and SMAD signaling both mediate the angiotensin II driven progression towards overt heart failure in homozygous TGR(mRen2)27. J Mol Med 82:678687

14. Lipsic E, van der Meer P, Henning RH, Suurmeijer AJ, Boddeus KM, van Veldhuisen DJ, van Gilst WH, Schoemaker RG (2004) Timing of erythropoietin treatment for cardioprotection in ischemia/ reperfusion. J Cardiovasc Pharmacol 44:473-479

15. van der Meer P, Lipsic E, Henning RH, Boddeus K, van der Velden J, Voors AA, van Veldhuisen DJ, van Gilst WH, Schoemaker RG (2005) Erythropoietin induces neovascularization and improves cardiac function in rats with heart failure after myocardial infarction. J Am Coll Cardiol 46:125-133

16. van der Meer P, Lipsic E, Henning RH, de Boer RA, Suurmeijer AJ, van Veldhuisen DJ, van Gilst WH (2004) Erythropoietin improves left ventricular function and coronary flow in an experimental model of ischemia-reperfusion injury. Eur J Heart Fail 6:853-859

17. Westenbrink BD, Lipsic $E$, van der Meer $P$, van der Harst $P$, Oeseburg H, Du Marchie Sarvaas GJ, Koster J, Voors AA, van Veldhuisen DJ, van Gilst WH et al (2007) Erythropoietin improves cardiac function through endothelial progenitor cell and vascular endothelial growth factor mediated neovascularization. Eur Heart J 28:2018-2027

18. Westenbrink BD, Ruifrok WP, Voors AA, Tilton RG, van Veldhuisen DJ, Schoemaker RG, van Gilst WH, de Boer RA (2010) Vascular endothelial growth factor is crucial for erythropoietin-induced improvement of cardiac function in heart failure. Cardiovasc Res 87:30-39

19. Westenbrink BD, Oeseburg H, Kleijn L, van der Harst P, Belonje AM, Voors AA, Schoemaker RG, de Boer RA, van Veldhuisen DJ, van Gilst WH (2008) Erythropoietin stimulates normal endothelial progenitor cell-mediated endothelial turnover, but attributes to neovascularization only in the presence of local ischemia. Cardiovasc Drugs Ther 22:265-274

20. Feng M, Whitesall S, Zhang Y, Beibel M, D’Alecy L, DiPetrillo K (2008) Validation of volume-pressure recording tail-cuff blood pressure measurements. Am J Hypertens 21:1288-1291

21. Pacher P, Nagayama T, Mukhopadhyay P, Batkai S, Kass DA (2008) Measurement of cardiac function using pressure-volume conductance catheter technique in mice and rats. Nat Protoc 3:1422-1434

22. Wang Y, Qian C, Roks AJ, Westermann D, Schumacher SM, Escher F, Schoemaker RG, Reudelhuber TL, van Gilst WH, Schultheiss HP et al (2010) Circulating rather than cardiac 
angiotensin-(1-7) stimulates cardioprotection after myocardial infarction. Circ Heart Fail 3:286-293

23. Kuipers I, van der Harst P, Kuipers F, van Genne L, Goris M, Lehtonen JY, van Veldhuisen DJ, van Gilst WH, de Boer RA (2010) Activation of liver X receptor-alpha reduces activation of the renal and cardiac renin-angiotensin-aldosterone system. Lab Invest 90:630-636

24. Vandesompele J, De Preter K, Pattyn F, Poppe B, Van Roy N, De Paepe A, Speleman F (2002) Accurate normalization of real-time quantitative RT-PCR data by geometric averaging of multiple internal control genes. Genome Biol 3: research0034

25. Ezekowitz JA, McAlister FA, Armstrong PW (2003) Anemia is common in heart failure and is associated with poor outcomes: insights from a cohort of 12065 patients with new-onset heart failure. Circulation 107:223-225

26. van der Meer P, Voors AA, Lipsic E, Smilde TD, van Gilst WH, van Veldhuisen DJ (2004) Prognostic value of plasma erythropoietin on mortality in patients with chronic heart failure. J Am Coll Cardiol 44:63-67
27. van der Meer P, Lok DJ, Januzzi JL, de la Porte PW, Lipsic E, van Wijngaarden J, Voors AA, van Gilst WH, van Veldhuisen DJ (2008) Adequacy of endogenous erythropoietin levels and mortality in anaemic heart failure patients. Eur Heart J 29:1510-1515

28. Belonje AM, Voors AA, van der Meer P, van Gilst WH, Jaarsma T, van Veldhuisen DJ (2010) Endogenous erythropoietin and outcome in heart failure. Circulation 121:245-251

29. Hansson A, Zetterblad J, van Duren C, Axelson H, Jonsson JI (2007) The Lim-only protein LMO2 acts as a positive regulator of erythroid differentiation. Biochem Biophys Res Commun 364:675-681

30. Kaneko H, Shimizu R, Yamamoto M (2010) GATA factor switching during erythroid differentiation. Curr Opin Hematol 17:163-168

31. Ohneda K, Yamamoto M (2002) Roles of hematopoietic transcription factors GATA-1 and GATA-2 in the development of red blood cell lineage. Acta Haematol 108:237-245

32. Jin F, Zhai Q, Qiu L, Meng H, Zou D, Wang Y, Li Q, Yu Z, Han J, Li Q et al (2008) Degradation of BM SDF-1 by MMP-9: the role in G-CSF-induced hematopoietic stem/progenitor cell mobilization. Bone Marrow Transplant 42:581-588 